\title{
Reproductive system and spermatozoa ultrastructure support the phylogenetic proximity of Megadasys and Crasiella (Gastrotricha, Macrodasyida)
}

\author{
Loretta Guidi $^{1,4}$, M. Antonio Todaro², Marco Ferraguti ${ }^{3}$, Maria Balsamo ${ }^{1}$ \\ ${ }^{1}$ Dipartimento di Scienze della Terra, della Vita e dell'Ambiente, Università di Urbino 'Carlo Bo', Italy \\ ${ }^{2}$ Dipartimento di Scienze della Vita, Università di Modena-Reggio Emilia, Modena, Italy \\ ${ }^{3}$ Dipartimento di Bioscienze, Università degli Studi di Milano, Via Celoria 26, I-20133 Milano, Italy \\ ${ }^{4}$ E-mail: loretta.guidi@uniurb.it
}

Key words: Cephalodasyidae, phylogeny, Planodasyidae, reproductive traits, spermatogenesis

\begin{abstract}
The reproductive system and the spermatozoon of Megadasys sterreri from Lanzarote (Canary Islands, Spain) were studied at structural and ultrastructural levels. The species is a simultaneous hermaphrodite with cross-fertilization and shows paired gonads, the male anterior and the female posterior, and both gametes mature in a caudo-cephalic direction. Sperm ducts converge on the midline and open into a ventral common pore. Two sexual accessory organs are present in the caudal trunk. A pipe-like frontal organ lies between the ovaries and the caudal organ, and is composed of a long, thin region connected to a large zone containing mature and degenerating spermatozoa. The cigar-like caudal organ is elongate, bulky and is made of an anterior glandulo-muscular region and a posterior muscular one. Spermatozoa are long, filiform cells formed by an acrosome, a nucleus-mitochondrial complex, and a flagellum. The long acrosome is composed of an apical twisted region and a basal straight region. The nucleo-mitochondrial complex is formed by a spring-shaped nucleus surrounding basally the mitochondrion and apically a granular material. The flagellum has a 9x2+2 axoneme, characterized by a dense and prominent central sheath surrounding the central tubules. Megadasys sterreri (Cephalodasyidae) shows the same reproductive layout as Crasiella (Planodasyidae): paired gonads, caudo-cephalic maturation of gametes, sperm ducts converging into a common ventral pore, and two sexual accessory organs. Also the spermatozoa ultrastructure shows two similarities in the two genera: a peculiar prominent central sheath in the axoneme and a similar structure of the basal region of the acrosome. Considering the likely polyphyletic nature of the family Cephalodasyidae, and the sister-taxon relationship of Megadasys and Crasiella that emerged from a recent molecular phylogenetic study, a close relationship between the two taxa appears to be very likely; consequently, we propose to remove Megadasys from the family Cephalodasyidae and affiliate it to the family Planodasyidae.
\end{abstract}

\section{Contents}

Introduction 119

Material and methods 123

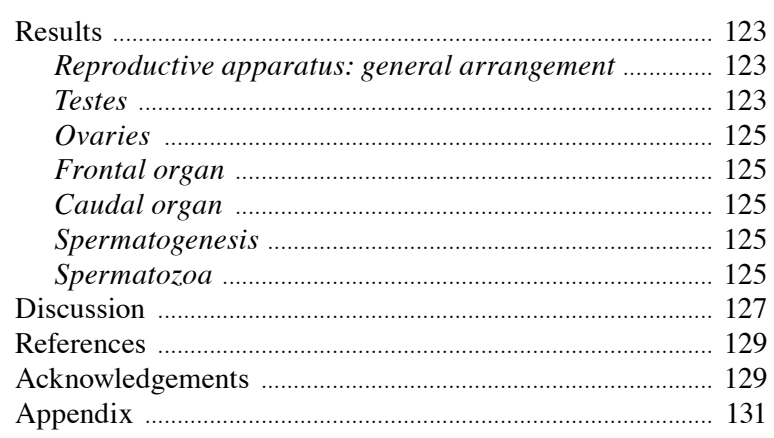

\section{Introduction}

The cosmopolitan phylum Gastrotricha includes about 790 microscopic, aquatic, meiobenthic species grouped into two orders: Macrodasyida and Chaetonotida. Macrodasyida include approximately 340 vermiform marine species, generally hermaphroditic with cross-fertilization. Chaetonotida include over 450 species, most of which are parthenogenetic and freshwater, with only some hermaphroditic and marine forms (Todaro and Hummon, 2008; Balsamo et al., 2009; Hummon and Todaro, 2010; Todaro et al., 2012; Kånneby et al., 2013). Current in-group systematization is mainly based on morphological grounds; however, recent phylogenetic studies have suggested that several taxa are non-monophyletic, with some contrasts between the evolutionary scenarios inferred from morphology or molecules (Hochberg and Litvaitis, 2000; Todaro et al., 2006, 2011, 2012 vs Kieneke et al., 2008; Kånneby et al., 2013).

A re-examination in an evolutionary perspective of known morphological characteristics along with the acquisition of new anatomical and biological data have permitted a re-systematization of some taxa (by establishing new families: Todaro et al., 2006, 2012; Leasi 


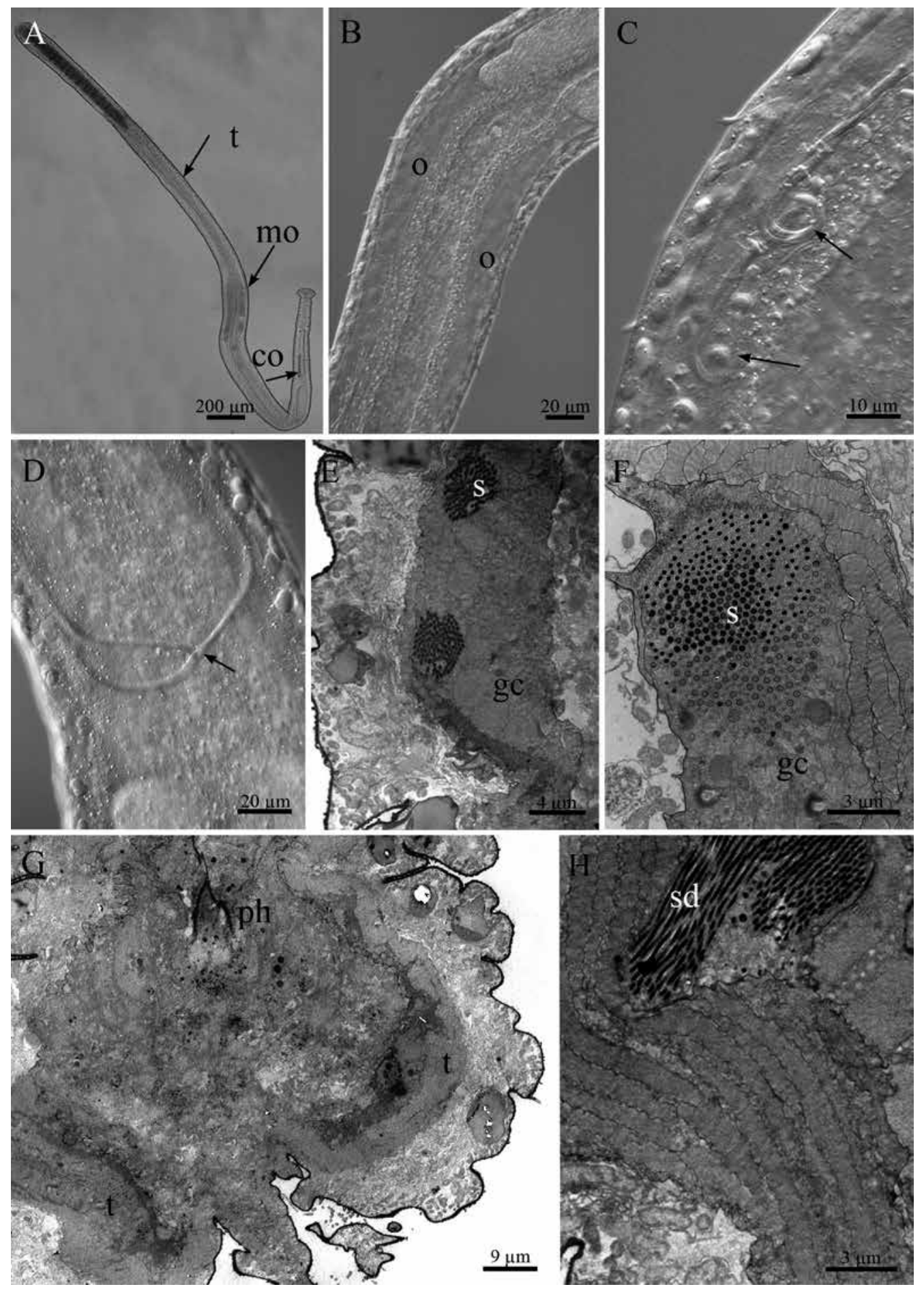

Fig. 1. Megadasys sterreri: habitus, testis and sperm ducts. A. Habitus of an adult specimen. The testis (t), the mature oocyte (mo) and the caudal organ (co) are visible. B. Portion of the body showing the two ovaries (o). C. The characteristic loops formed by sperm ducts (arrows). D. Merging of sperm ducts (arrow). E. Longitudinal section of the body showing the compact testis in which the germinal cells (gc) mature in a caudo-cephalic direction and the mature spermatozoa (s) migrate ventro-laterally along each testis moving to its posterior end. F. Cross-section of the body showing the compact testis: note the germinal cells (gc) and the mature spermatozoa (s). G. Oblique section of the testes $(\mathrm{t})$ at the level of the pharynx (ph). H. Sperm duct (sd) in a 'loop'. 
and Todaro, 2008; Hummon and Todaro, 2010), reducing phylogenetic conflicts and/or indicating the hypotheses based on molecular markers to be the most robust (compare position of Redudasys in Kieneke et al., 2008 vs Todaro et al., 2012). While several uncertainties still remain, it is interesting noting that where phylogenetic groupings based on morphology and on molecules agree with the current systematization the representatives of that taxon/grouping share homolo- gies in the reproductive traits, especially at family level. For instance, members of the Thaumastodermatidae as well as of the Turbanellidae are characterized by peculiarities of their reproductive system and/or gamete ultrastructure (Balsamo et al., 2002; Marotta et al., 2005, 2011; Todaro et al., 2011), and these characteristics provide a strong basis for monophyly, that is also supported in all phylogenetic analyses (Hochberg and Litvaitis, 2000; Todaro et al., 2003,
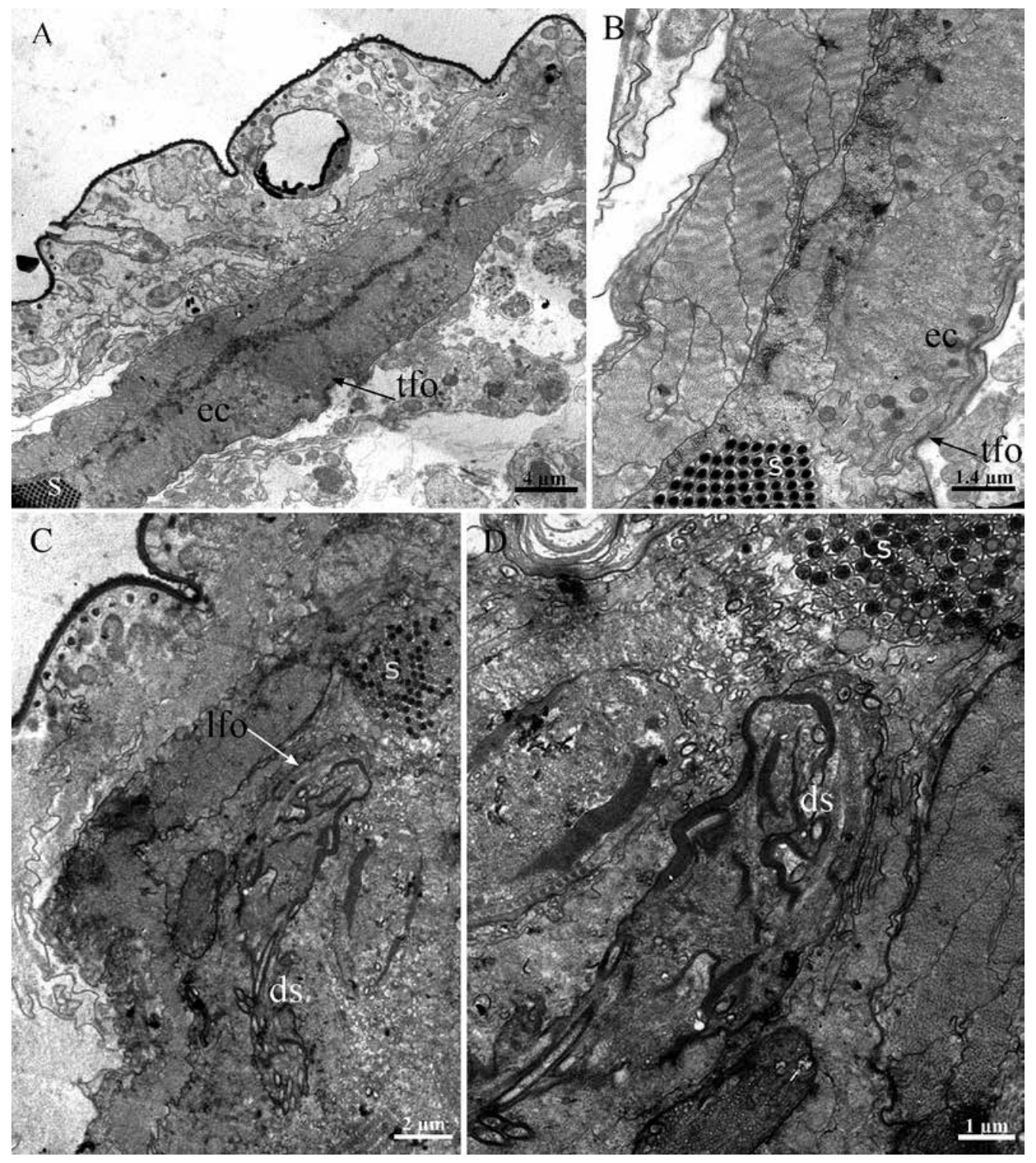

Fig. 2. Megadasys sterreri: frontal organ. A-B. Longitudinal section of the frontal organ thin portion (tfo): note the epithelial cells (ec) and mature spermatozoa (s). C-D Longitudinal section of the large portion of the frontal organ (lfo). Degenerating (ds) and non-degenerating spermatozoa $(\mathrm{s})$ are visible. 

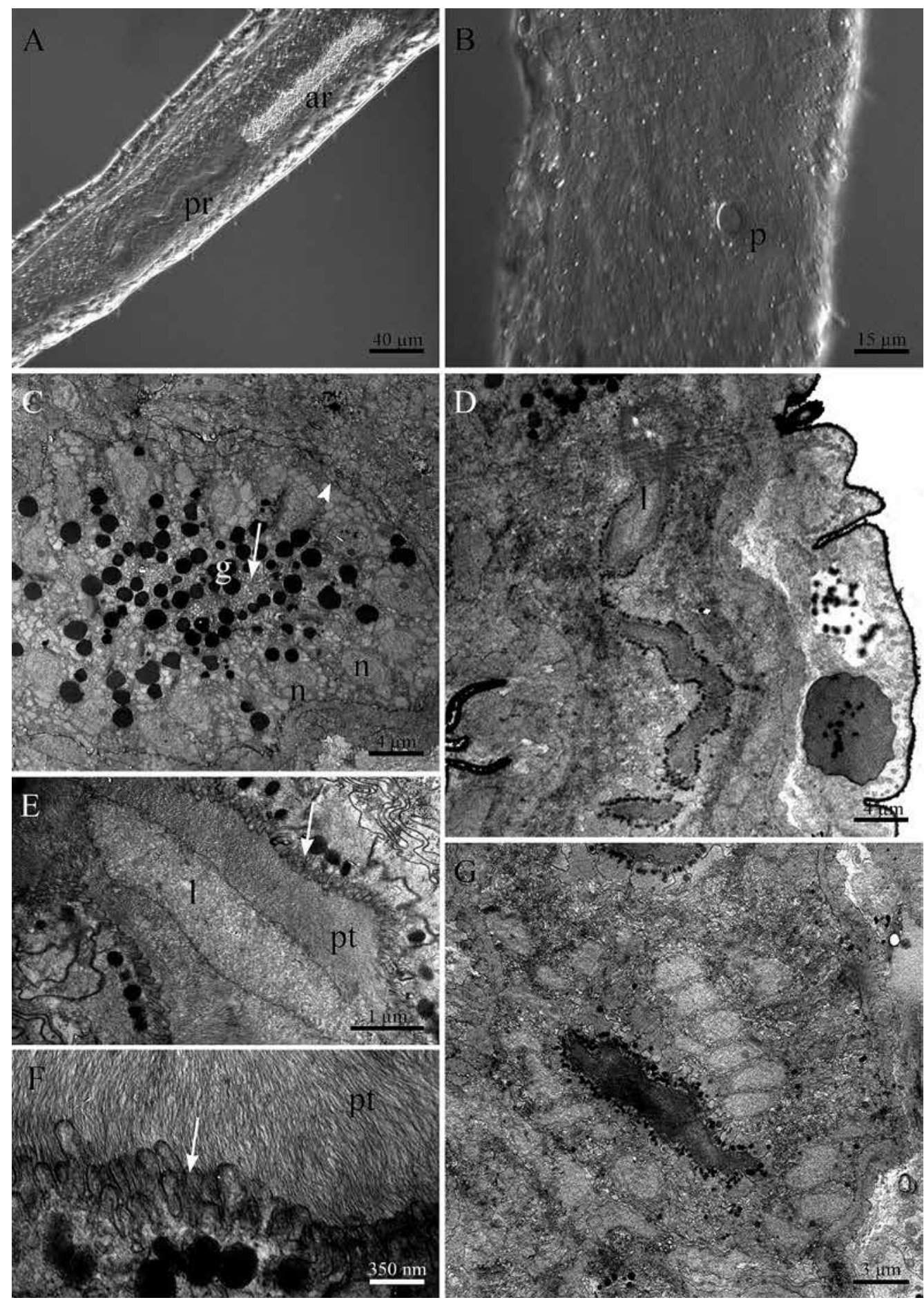

Fig. 3. Megadasys sterreri: caudal organ. A-B. The musculo-glandular caudal organ is long and cylindrical and formed by two regions clearly recognizable: the anterior (ar) and the posterior region (pr). The latter has a large lumen that opens in a terminal pore (p). C. Cross-section of anterior region of caudal organ: note the basal position of the nuclei (n) of the glandular cells, their inner secretion granules (g), and microvilli (arrow) projecting out into the virtual lumen. The thick basal lamina surrounding circular fibers is clearly visible (arrowhead). D-E-F. Posterior part of caudal organ: note the large lumen (l), the microvilli (arrow) and the network of glycoproteins $(\mathrm{pt})$. G. The terminal region of the caudal organ near to the pore. 
2006, 2011, 2012; Petrov et al., 2007; Paps and Riutort, 2012). This highlights the strong phylogenetic signal embedded in the reproductive traits as well as their possible utility in the process of natural systematization of Gastrotricha. Moreover, it calls for an indepth comparison of reproductive structures and gametes of species that are currently ascribed to different families, despite the fact that molecular data show them combined into a common clade. One such a case is represented by the species of Crasiella and Megadasys, two genera that are currently assigned to the families Planodasyidae Rao and Clausen, 1970 and Cephalodasyidae Hummon and Todaro, 2010, respectively, but are likely to form a monophylum based on the 18S rDNA gene (Todaro et al., 2012, see also Paps and Riutort, 2012). While there is already a satisfactory information on the reproductive system of two species of Crasiella (Guidi et al., 2011), much still remains to be learned about the structure of the reproductive apparatus and male gametes in species of $\mathrm{Me}$ gadasys. Here, we supply additional data on Megadasys sterreri (Boaden, 1974) with the aim of explaining the current systematization of the genus.

\section{Material and methods}

Sand samples of Megadasys sterreri (Boaden, 1974) were collected in October 2011 from 11-20 m water depth at Mala, Lanzarote (Canary Islands, Spain; Riera and Todaro, 2012). Gastrotrichs were extracted from the sediment by the narcotization-decantation technique, using an isosmotic (7\%) magnesium chloride solution (Giere, 2009). The fauna-containing supernatant was then poured directly into a $5-\mathrm{cm}$ in diameter Petri dish and scanned for specimens under a Wild M3 dissecting microscope. For light microscopy (Todara and Hummon, 2008), three adult specimens of Megadasys sterreri were removed with a micropipette from the Petri dish, fresh-mounted on slides and observed under a Leitz Dialux 20 microscope equipped with Differential Interference Contrast (Nomarski). During observation, the animals were photographed with a DS-Fi1 Nikon digital camera and measured with the Nikon NIS-F software.

For transmission electron microscopy, six mature specimens were fixed overnight $(24 \mathrm{~h})$ in $2 \%$ glutaraldehyde in a $0.1 \mathrm{M}$ sodium cacodylate buffer ( $\mathrm{pH}$ 7.4), and stored in a $0.1 \mathrm{M}$ sodium cacodylate buffer. They were then post-fixed in $1 \%$ osmium tetroxide in the same buffer, repeatedly washed in the same buffer, dehydrated in a graded ethanol series and embedded in Araldite. Semi-thin ( $2 \mu \mathrm{m}$ thick) and ultra-thin sections (70 nm thick) were cut with an LKB Ultrotome $2088 \mathrm{~V}$. The semi-thin sections were stained with toluidine blue while the ultra-thin sections were contrasted with a saturated solution of uranyl acetate in ethanol $50 \%$, followed by lead citrate solution (Reynolds, 1963). The ultra-thin sections were observed under a Philips CM10 transmission electron microscope. The locations of some morphological characteristics along the body are given in percentages of units (U) of the total body length, measured from the anterior to the posterior.

\section{Results}

\section{Reproductive apparatus: general arrangement}

Mature specimens of Megadasys sterreri are simultaneous hermaphrodites. Two band-shaped testes lie at the sides of the anterior intestine; the sperm ducts are quite long and sinuous and form 2-4 characteristic 'loops' over their length. They originate at about the mid-testis and extend posteriorly to about mid body where they fuse on the mid-ventral plane and open exteriorly through a single median pore (Fig. 1A, C-D). Two ovaries are lateral to the posterior intestine and join mid-dorsally anteriorly; a large mature egg, dorsal to the intestine is usually visible at about mid body (Fig. 1A-B). In the caudal trunk region an elongate, bulky, musculo-glandular organ interpreted as a caudal organ is present (Fig. 1A). An epithelial frontal organ lies between the ovaries and the caudal organ.

\section{Testes}

The two testes are thin and compact bands extending from the level of the pharyngeo-intestinal junction (U24) to U44. The germinal epithelium is arranged peripherally along the entire length of each testis, and gametes mature in a caudo-cephalic direction: mature spermatozoa migrate caudally from the most anterior region of the testis along its external side. A testis lumen is not present (Fig. 1E-G). The sperm ducts begin at about mid-testis, extend posteriorly, and fuse on the middle ventral plane in the trunk region. The common sperm duct continues as a short, slightly wider canal that is usually oriented towards the left body side. The male pore was not clearly detected so it is uncertain whether it opens at the confluence of the sperm ducts or at the end of the common canal (Fig. 1C-D, H). 

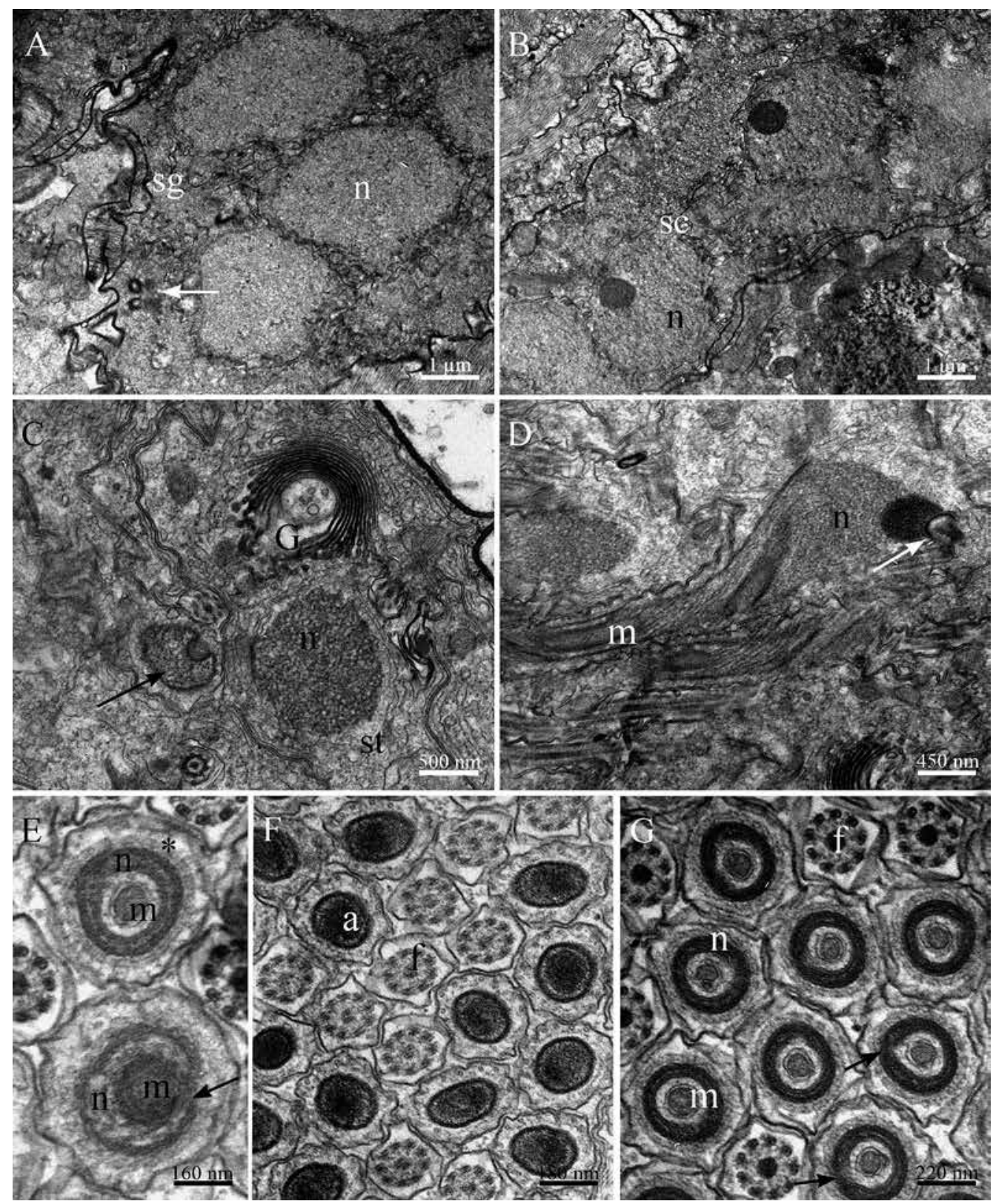

Fig. 4. Megadasys sterreri: spermatogenesis. A. Spermatogonia (sg) in mitotic division (arrow) showing large nuclei (n) with regularly distributed chromatin. B. Spermatocytes (sc) showing large nuclei (n) with nucleolus and chromatin condensed in small patches. C. On the right of the picture note an early spermatid (st) with a big roundish nucleus (n) and a well-developed Golgi apparatus (G). On the left a mid-spermatid in cross-section is visible: note the nucleus forming a large "C" around the mitochondrion (arrow). D. Mid-spermatids: note the mitochondrion (m) entering in the nucleus (n) and the conical piece lying in the nuclear base (arrow). E. Cross-sections of a mid (arrow) and a late spermatid (*): in the first the nucleus surrounds the mitochondrion forming a large ' $\mathrm{C}$ ', in the second the nucleus forms a ring. F-G. U-shaped spermatids: at this stage flagella are parallely arranged to the nuclei and acrosomes so in cross-section can be seen only flagella (f) and acrosome (a) or flagella and nuclei/mitochondria (n/m). The arrows show the zone where the nucleus is closing on itself. 


\section{Ovaries}

Two lateral ovaries extend along the posterior intestine from U53 to U73. In mature specimens, at least 7-8 oocytes are present on each side of the body: they are arranged in a line and the most anterior one is the already mentioned mature egg that often appears, horse-shoe shaped, surrounding the middle intestine dorsally. Its size may reach $330 \mu \mathrm{m}$ in length and $38 \mu \mathrm{m}$ in width (Fig. 1A-B).

\section{Frontal organ}

An elongate, pipe-like frontal organ, parallel to the main body axis, is present between U76 and U86. It is formed by an anterior long and thin epithelial part (100 $\mu \mathrm{m}$ long and $8 \mu \mathrm{m}$ wide) surrounding a lumen in which a bundle of mature spermatozoa is generally visible, likely in transit to the mature oocyte. The epithelial cells contain round vacuoles with medium-dense granular content, which may have nutritive or enzymatic functions. This part continues into a posterior large zone where few mature and many degenerating spermatozoa are present. The surface of the whole organ is in touch with the body musculature (Fig. 2A-D).

\section{Caudal organ}

The caudal organ is a cigar-like structure $(280 \mu \mathrm{m}$ long and $40 \mu \mathrm{m}$ wide) that extends from U87 to U95. It is composed of two parts of similar length: an anterior glandulo-muscular region (130 $\mu \mathrm{m}$ long) and a posterior muscular region (150 $\mu \mathrm{m}$ in length), opening outside through a terminal pore (Fig. 3A-B). The musculature of both regions is composed only of circular fibers and is completely surrounded by a thick basal lamina (Fig. $3 \mathrm{C}-\mathrm{D})$. The anterior wall of the organ is formed by large cells arranged in a single layer. These cells contain a lot of secretory granules, and appear structurally and functionally polarized, with the nucleus close to the cell side that faces the surrounding musculature and much cytoplasm condensed along the cell side facing the interior of the organ. The latter shows numerous microvilli that extend to touch those of the other cells (Fig. 3C). The posterior region of the organ appears hollow by the presence of a sinuous duct (Fig. 3A, $\mathrm{D}-\mathrm{G})$, the lumen of which is rather wide and lined for its whole length by columnar epithelial cells with numerous, stout microvilli, covered with a thick (200 $\mathrm{nm}$ ), filamentous layer made of a network of membrane-bound polymeric material (glycoproteins) (Fig. 3E-
F). Near the microvilli a large number of packed secretion cytoplasmic granules is visible, round or ovoid in shape, homogeneous and very electron-dense (Fig. 3D-E, G).

\section{Spermatogenesis}

Spermatogonia are characterized by a large ovoidal nucleus with granular and dispersed chromatin. They have little cytoplasm containing several mitochondria, many free ribosomes and duplicated diplosomes (Fig. 4A). Spermatocytes are large, round cells with a circular nucleus with a nucleolus (Fig. 4B). In early spermatids, the nuclear chromatin progressively condenses forming a large, round nucleus immersed in an organelle-rich cytoplasm. The Golgi apparatus is well developed and produces a-high number of vesicles full of electron-dense material. The flagellum is already present (Fig. 4C). In the mid-spermatids, the nucleus starts elongating and at the same time a long mitochondrion, arising from the fusion of many smaller mitochondria, sticks to the nuclear envelope and gradually sinks into the nucleus, that in cross-section initially takes the form of a large 'C' (Fig. 4D-E). The acrosome starts to develop when the vesicles produced by the Golgi apparatus progressively condense and organize in a pile. While the nuclear-acrosomal complex is growing, the axoneme starts lengthening parallel to the nucleus (U-shaped spermatids in Fig. 4D). Late spermatids have completed the nuclear elongation and their aspect is similar to that of mature spermatozoa except for the cell U-shape and for the manchette of microtubules surrounding both the nucleus and the acrosome. At this stage the nucleus completely surrounds the mitochondrion (Fig. 4F,G). The terminal spermatids start sliding along the external side of each testis in a caudal direction (Fig. 1D). Spermatids complete the maturation by rotating the flagellum which assumes its final position in line with the nucleus-acrosome complex. Mature spermatozoa migrate into the sperm ducts, up to converge in the single ventral male pore.

\section{Spermatozoa}

The spermatozoon of Megadasys sterreri is a long (about 125-130 $\mu \mathrm{m}$ ), filiform cell made up of an anterior acrosome, a nucleus-mitochondrial complex, and a tail. The apical portion, composed of the acrosome and the nucleus, is about $45-50 \mu \mathrm{m}$ long. The acrosome is long and sub-divided into two different morphological regions (Fig. 5K). The apical region is a twisted 

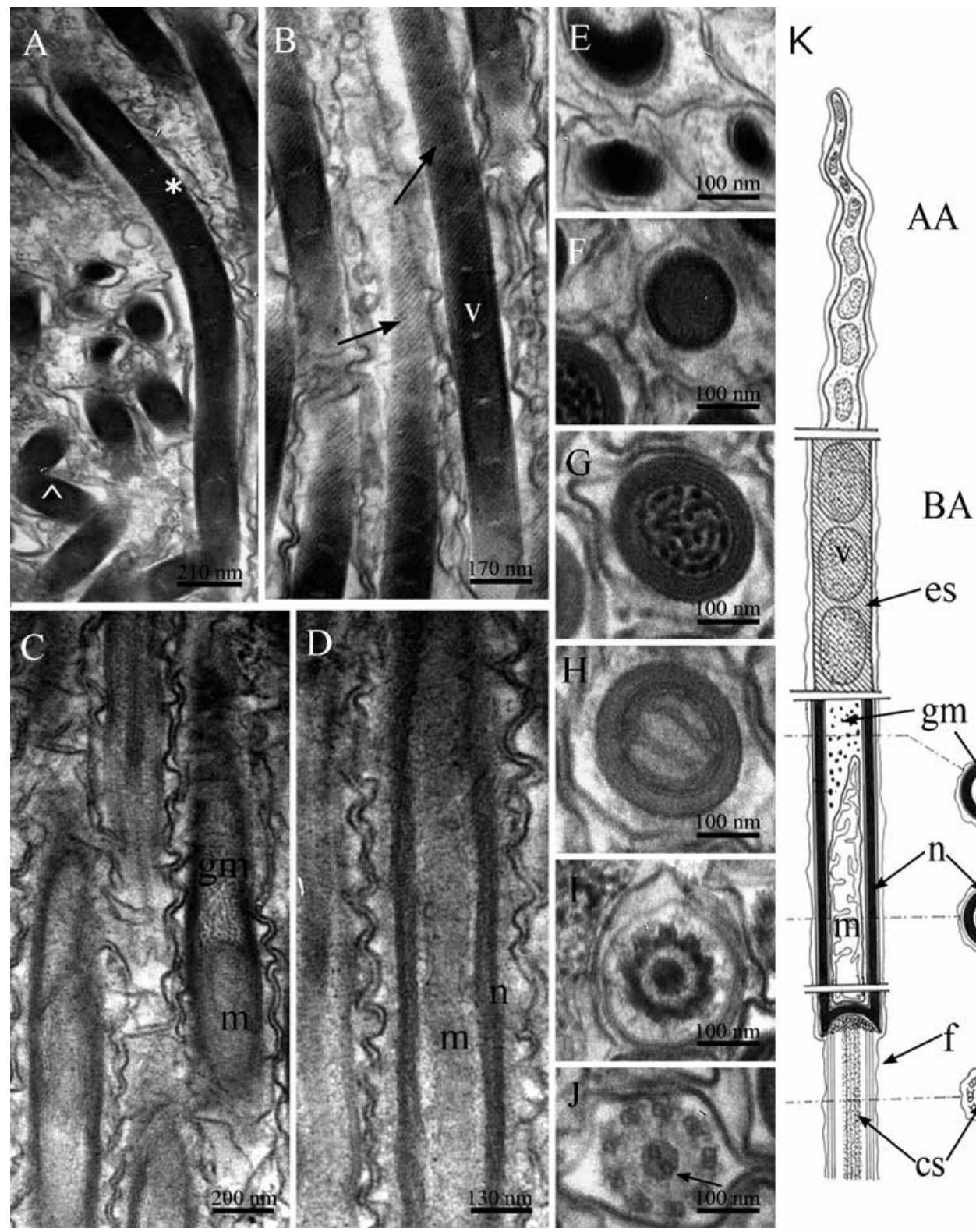

BA

Fig. 5. Megadasys sterreri: spermatozoa. A. Longitudinal sections of acrosome: the apical twisted $(\wedge)$ and the basal straight $(*)$ regions are visible. B. Longitudinal section of the basal region of the acrosome: note the external striation (arrows) and the piled oval vesicles (v). C. Longitudinal section showing the region where the nucleus (n) surrounds the granular material (gm) and the mitochondrion (m). D. Portion of the nucleus/mitochondrion complex $(\mathrm{n} / \mathrm{m})$. E. Cross-section of the apical twisted region of the acrosome. F. Cross-section of the basal straight region of the acrosome. G. Cross-section of the nucleus surrounding the granular material. H. Cross-section of the nucleus/mitochondrion complex. I. Cross-section of the conical piece connecting the flagellum to the nucleus. J. Cross-section of the flagellum: note the prominent central sheat (arrow). K. Schematic drawing of the mature spermatozoon of Megadasys sterreri showing the apical acrosomal region (AA), the basal acrosomal region (BA), oval piled vesicles inside the acrosome (v), the external striation (es), the nucleus (n), the mitochondrion (m) and the granular material (gm) into the nucleus, the flagellum (f) and the central sheat (cs). 
tube with an inner structure made of piled oval vesicles (Fig. 5A, E, K); its diameter increases in a posterior direction from 100 to $200 \mathrm{~nm}$. The basal region is a straight tube with a constant diameter of $200 \mathrm{~nm}$ containing oval vesicles similar in shape to those of the anterior region but with a larger diameter $(150 \mathrm{~nm}$, Fig. $5 \mathrm{~A}-\mathrm{B}, \mathrm{F}, \mathrm{K}$ ); it is surrounded by a sheath showing an evident oblique striation (Fig. 5B, K). The nucleo-mitochondrial portion is about $15-20 \mu \mathrm{m}$ long: anteriorly the spring-shaped nucleus surrounds a granular material devoid of mitochondria (230 $\mathrm{nm}$ in diameter), while posteriorly the mitochondrion is surrounded by the nucleus, with a diameter of $300 \mathrm{~nm}$ (Figs. 5C-D, G-H, $\mathrm{K})$. The flagellum is $80 \mu \mathrm{m}$ long and has a 9x2+2 axoneme, characterized by a dense and prominent central sheath surrounding the central tubules (Fig. 5J, K). The nucleus and the flagellum are connected by a conical piece lying in a groove at the nuclear base (Fig. $4 \mathrm{D}, 5 \mathrm{I})$.

\section{Discussion}

The general layout of the reproductive apparatus of the specimens from Lanzarote matches the description of the reproductive system of specimens of Megadasys sterreri from Ireland provided by Boaden (1974) and also those of Megadasys minor from Roscoff described by Kisielewski (1987). The layout is also in accordance with our unpublished observations on various species of Megadasys from other areas of the World (i.e. Mediterranean Sea, North Sea, Caribbean Sea and Persian Gulf; M.A. Todaro, unpubl. data). Therefore, the apparent absence of a frontal organ in Megadasys pacificus (Schmidt, 1974) should be considered either as an autoapomorphy or an oversight by the author. Considering that the size of this organ may vary across species of the same genus, and that it was difficult to discern it also in our specimens even under DIC optics, the latter hypothesis seems the most likely. Consequently, it may be said that members of the genus Megadasys are hermaphrodite, with paired and separate male and female gonads, with a single male pore and both frontal and the caudal organs. The present ultrastructural investigation has shown that both male and female gametes mature in a posterior to anterior direction.

All the genera of the family Cephalodasyidae ( $\mathrm{Ce}$ phalodasys, Dolichodasys, Megadasys, Mesodasys, Paradasys, Pleurodasys) have paired gonads but may differ in $i$ ) relative position of male and female gonads along the body; $i$ ) direction of development of male and female germ cells; iii) number of the male pores, and $i v$ ) occurrence of accessory sexual organs.

Differences in the structure of the reproductive system of species of Cephalodasyidae appear so marked that it is hard to find potential synapomorphies that justify their grouping into a single family. For example, species of Mesodasys bear bilateral male and female gonads, with anterior testes and posterior ovaries, and both types of germ cells develop in a frontward direction. Mesodasys gastrotrichs are unique in the family in that their sperm ducts are directly connected to the caudal organ; yet a frontal organ is missing and hypodermic impregnation likely occurs. The sperm of an individual may be injected inside different regions of the partner's body (Ruppert, 1991; Fregni et al., 1999)

Ruppert and Shaw (1977) describe members of Dolichodasys as possessing a pair of bivalent gonads (ovariotestis) that produce female germ cells anteriorly and male germ cells posteriorly, with both cell types maturing in a caudal direction, yet the sperm ducts open to the exterior with independent pores. Furthermore, in Dolichodasys the frontal and caudal organs are functionally distinct units but are connected by a compact tissue band; the frontal organ receives allosperm by means of a ventral pore while an internal pore ensures that these sperm reach the mature eggs. The caudal organ is structurally complex as it possesses two canals intercommunicating both distally and proximally with a single external pore, which allows the intake and extrusion of the autosperm. Members of Cephalodasys (except for $C$. hadrosomus for which no data are available) possess bilateral male and female gonads, anterior testes and posterior ovaries, with the two types of germ cells maturing in opposite directions i.e. sperm from posterior to anterior and eggs from anterior to posterior. Sperm ducts join ventrally at the mid line and open externally into a common pore. In species for which the accessory sexual organs have been reported, the frontal organ is always present while the caudal organ may be absent (e.g. C. pacificus and C.palavensis).

Information on the reproductive system of Pleurodasys and Paradasys are particularly poor and should be taken with caution. According to the data in the literature, members of both taxa possess paired gonads with anterior testes and posterior ovaries; both germ cells mature in a frontward direction (Boaden, 1963; Rao, 1980, see also Ruppert and Shaw, 1977). Furthermore, sperm ducts open separately in Pleurodasys species whereas no information are available for Paradasys species. Likewise, no accessory reproductive organs have been reported for Paradasys while a 'bursa 
seminalis' has been indicated for Pleurodasys (see Boaden, 1963). It should be emphasized that the correspondence of the 'bursa' to the frontal or to the caudal organ may be problematic as in the past this term has been applied to both the organ with female function (frontal organ) and to the organ with male function (caudal organ) (see Ruppert, 1991). Based on information derived from an undescribed species of Pleurodasys from South Africa, in which a structure resembling a frontal organ is present (M.A. Todaro, unpubl. data), the correspondence between the bursa described by Boaden (1963) and the frontal organ reported in other Macrodasyida appears as the most likely scenario.

From all the examples reported above, it appears clear that the structure of the reproductive system of Megadasys finds no match in any of the other genera of Cephalodasyidae; furthermore, differences exist also among the other genera of the family casting doubts on its monophyly.

The family Cephalodasyidae derives from a recent splitting of the family Lepidodasyidae and was established mainly to accommodate the genera clearly different from the type-genus Lepidodasys (Hummon and Todaro, 2010). At that time, clear autoapomorphies such as the presence and ultrastructure of the cuticular scales, pharynx organization and sperm ultrastructure provided solid grounds for separating Lepidodasys from the other genera of the family, for which however no synapomorphies could be detected (Ruppert, 1991; Guidi et al.,2004; for peculiarity in the muscular system see Hochberg et al., 2013).

A recent phylogenetic analysis based on the gene $18 \mathrm{~S}$ rDNA has found Megadasys allied with Crasiella (Todaro et al., 2012); while at first sight this novel hypothesis may appear provocative, it is worthy of consideration especially in the light of the comparison of the reproductive systems of the two taxa, from which a perfect match in their layout emerges.

In fact, representatives of Crasiella, like the specimens of Megadasys, $i$ ) possess paired gonads, anterior male and posterior female, $i$ ) have both germ cells maturing from posterior to anterior, iii) sperm ducts converge ventrally on the midline and open to the exterior through a common pore, and $i v$ ) possess both frontal and caudal organs (cf. Guidi et al., 2011 and this study).

While components and arrangement of the reproductive systems of Megadasys and Crasiella are the same and strongly allude to a common origin of this trait as a whole, the specific shape and structure of the organs composing the two reproductive systems may be taxon specific. However, possible observations on additional species may help in assigning the specificity of a trait at the level of species or genus. Likewise, an overemphasis on singular reproductive characters may incorrectly align Megadasys or Crasiella more to other macrodasyidan taxa than to each other. For example, a caudal organ showing a single internal canal and a single external pore aligns Megadasys to Macrodasys more than it does Megadasys to Crasiella; on the other hand, the two-canal caudal organ of Crasiella is more similar to that of Dolichodasys than to Megadasys. However, the presence of a single type of musculature (circular) in the caudal organ of Megadasys vs two types (circular and longitudinal) in Macrodasys and the way the two canals are interconnected to each other in the caudal organ of Crasiella vs Dolichodasys reveals the limits of the resemblance between these organs.

The spermatozoa ultrastructure of Megadasys sterreri provides additional support to the hypothesis of a phylogenetic alliance between Megadasys and Crasiella. In particular, the male gametes of Megadasys sterreri as those of Crasiella spp. possess an axoneme that has a peculiar modification of the central apparatus i.e. a prominent central sheath, which is lacking in all the other gastrotrich species studied so far. While the functional and evolutionary significance of this structure, which was first reported for species of Clitellata (Annelida) (see Ferraguti, 1984), has yet to be assessed, it clearly differentiates the spermatozoa of Megadasys and Crasiella from those of the other gastrotrichs. An additional spermatological trait in common between Megadasys and Crasiella is the structure of the basal region of the acrosome arranged as a straight tube with piled large oval vesicles. Moreover, both Megadasys and Crasiella lack the periaxonemal striated cylinder that is present in all the other species of Cephalodasyidae studied to date (Cephalodasys, Mesodasys spp.), making Megadasys the only genus of the family without a striated cylinder.

The same can be said of the oblique striation on the sheath involving the basal portion of the acrosome, a character shared with the species examined so far of the family Turbanellidae, and about the fine organization of the testis, which in Megadasys under TEM appears compact i.e. lacking an inner lumen, a characteristic shared only by Lepidodasys sp. and Urodasys anorektoxys among the numerous macrodasyidan species examined so far (Guidi et al., 2004; Balsamo et al., 2007).

To summarize, our study highligths that the general layout of the reproductive apparatus of Megadasys is 
more similar to that of Crasiella than to any other genus currently included in the family Cephalodasyidae, and moreover, the spermatozoa of the two species share some characters that are likely to be homologous. Thus, considering the clues pointing towards the potential polyphyletic nature of the family Cephalodasyidae and the sister-taxon relationships between Megadasys and Crasiella emerged from single gene studies (Paps and Riutort, 2012; Todaro et al., 2012) we consider the close relationship between Megadasys and Crasiella to be very likely. Consequently, we propose the genus Megadasys to be removed from the Cephalodasyidae and provisionally affiliated to the family Planodasyidae. Future morphological studies that fill the gap about the complete organization of the reproductive apparatus of species of the rare genus Planodasys (Rao and Clausen, 1970) and/or molecular phylogenetic analyses including this genus will confirm or disprove our hypothesis.

\section{Acknowledgements}

We would like to thank the two anonymous referees for their helpful comments.

\section{References}

Balsamo M, Ferraguti M, Guidi L, Todaro MA, Tongiorgi P. 2002. Reproductive system and spermatozoa of Paraturbanella tessieri (Gastrotricha, Macrodasyida): implications for the sperm transfer modality in Turbanellidae. Zoomorphology 121: 235-241.

Balsamo M, Guidi L, Pierboni L, Marotta R, Todaro MA, Ferraguti M. 2007. Living without mitochondria: spermatozoa and spermatogenesis in two species of Urodasys (Gastrotricha, Macrodasyida) from dysoxic sediments. Invertebrate Biology 126: 1-9.

Balsamo M, d'Hondt JL, Pierboni L, Grilli P. 2009. Taxonomic and nomenclatural notes on freshwater Gastrotricha. Zootaxa 2158: 1-19.

Boaden PJS. 1963. The interstitial fauna of some north Wales beaches. Journal of the Marine Biological Association of the United Kingdom 43: 79-96.

Boaden PJS. 1974. Three new thiobiotic Gastrotricha. Cahiers de Biologie Marine 15: 367-378.

Clausen C. 1968. Crasiella diplura gen. et sp. n. (Gastrotricha, Macrodasyoidea). Sarsia 33: 59-63

Ferraguti M. 1984. The comparative ultrastructure of sperm flagella central sheath in Clitellata reveals a new autapomorphy of the group. Zoologica Scripta 13: 201-207.

Fregni E, Balsamo M, Ferraguti M. 1999. Morphology of the reproductive system and spermatozoa of Mesodasys adenotubulatus (Gastrotricha, Macrodasyida). Marine Biology 135: 515-520.
Giere O. 2009. Meiobenthology: the microscopic motile fauna of aquatic sediments, 2nd ed. Berlin Heidelberg: SpringerVerlag.

Guidi L, Pierboni L, Ferraguti M, Todaro MA, Balsamo M. 2004. Spermatology of the genus Lepidodasys Remane, 1926 (Gastrotricha, Macrodasyida): towards a revision of the family Lepidodasyidae Remane, 1927. Acta Zoologica 85: 211-221.

Guidi L, Todaro MA, Ferraguti M, Balsamo M. 2011. Reproductive system of the genus Crasiella (Gastrotricha, Macrodasyida). Helgoland Marine Research 65: 175-185.

Hochberg R, Atherton S, Gross V. 2013. A new species of sublittoral marine gastrotrich, Lepidodasys ligni sp. n. (Macrodasyida, Lepidodasyidae), from the Atlantic coast of Florida. ZooKeys 289: 1-12.

Hochberg R, Litvaitis MK. 2000. Macrodasyida (Gastrotricha): A cladistic analysis of morphology. Invertebrate Biology 120: 124-135.

Hummon WD, Todaro MA. 2010. Analytic taxonomy and notes on marine, brackish-water and estuarine Gastrotricha. Zootaxa 2392: 1-32.

Kånneby T, Todaro MA, Jondelius U. 2013. Phylogeny of Chaetonotidae and other Paucitubulatina (Gastrotricha: Chaetonotida) and the colonization of aquatic ecosystems. Zoologica Scripta 42: 88-105.

Kieneke A, Rieman O, Ahlrichs WH. 2008. Novel implications for the basal internal relationships of Gastrotricha revealed by an analysis of morphological characters. Zoologica Scripta 37: 429-460.

Kisielewski J. 1987. New records of marine Gastrotricha from the French coasts of Manche and Atlantic. I. Macrodasyida, with descriptions of seven new species. Bulletin du Muséum national d'Histoire naturelle 9A: 837-877.

Leasi F, Todaro MA. 2008. The muscular system of Musellifer delamarei (Renaud-Mornant, 1968) and other chaetonotidans with implications for the phylogeny and systematization of the Paucitubulatina (Gastrotricha). Biological Journal of the Linnean Society 94: 379-398.

Marotta R, Guidi L, Pierboni L, Ferraguti M, Todaro MA, Balsamo M. 2005. Sperm ultrastructure of Macrodasys caudatus (Gastrotricha: Macrodasyida) and a sperm based phylogenetic analysis of Gastrotricha. Meiofauna Marina 14: 9-21.

Marotta R, Balsamo M, Ferraguti M, Fondello C, Guidi L, Todaro MA. 2011. The spermatozoon of Thaumastoderma moebjer$g i$ with the description of the sperm model for the family Thaumastodermatidae (Gastrotricha, Macrodasyida). Meiofauna Marina 19: 89-95.

Paps J, Riutort M. 2012. Molecular phylogeny of the phylum Gastrotricha: new data brings together molecules and morphology. Molecular Phylogenetics and Evolution 63: 208-212.

Petrov NB, Pegova AN, Manylov OG, Vladychenskaya NS, Mugue NS and Aleshin VV. 2007. Molecular phylogeny of Gastrotricha on the basis of a comparison of the 18S rRNA genes: Rejection of the hypothesis of a relationship between Gastrotricha and Nematoda. Molecular Biology 41: 445-452.

Rao GC. 1980. On a new species of Paradasys (Gastrotricha: Lepidodasyidae) from Andhra coast, India. Bulletin of the Zoological Survey of India 2: 213-215.

Rao GC, Clausen C. 1970. Planodasys marginalis gen. et sp. nov. and Planodasyidae fam. nov. (Gastrotricha Macrodasyoidea). Sarsia 42: 73-82. 
Reynolds ES. 1963. The use of lead citrate at high pH as electron-opaque stain in electron microscopy. Journal of Cell Biology 17: 208-212.

Riera R, Todaro MA. 2012. Check list of gastrotrichs from the Canary Islands. Revista de la Academia Canaria de Ciencias 24: 81-88.

Ruppert EE. 1991. Gastrotricha. Pp. 41-109 in: Harrison FW, Ruppert EE, eds, Microscopic Anatomy of Invertebrates, 4, Aschelminthes. New York: Wiley.

Ruppert EE, Shaw K. 1977. The reproductive system of gastrotrichs. I. Introduction with morphological data for two new Dolichodasys species. Zoologica Scripta 6: 185-195.

Schmidt P. 1974. Interstitielle Fauna von Galapagos. IV. Gastrotricha. Mikrofauna des Meeresbodens 26: 1-76.

Todaro MA, Hummon WD. 2008. An overview and a dichotomous key to genera of the phylum Gastrotricha. Meiofauna Marina 16: 3-20.

Todaro MA, Littlewood DTJ, Balsamo M, Herniou EA, Cassanelli S, Manicardi G, Wirz A, Tongiorgi P. 2003. The interrelationships of the Gastrotricha using nuclear small rRNA subunit sequence data, with an interpretation based on morphology. Zoologischer Anzeiger 242: 145-156.

Todaro MA, Telford MJ, Lockyer AE, Littlewood DTJ. 2006. Interrelationships of the Gastrotricha and their place among the Metazoa inferred from 18S rRNA genes. Zoologica Scripta 35: 251-259.

Todaro MA, Kånneby T, Dal Zotto M, Jondelius U. 2011. Phylogeny of Thaumastodermatidae (Gastrotricha: Macrodasyida) inferred from nuclear and mitochondrial sequence data. PLOS ONE 6: e17892.

Todaro MA, Dal Zotto M, Jondelius U, Hochberg R, Hummon WD, Kånneby T, Rocha CEF. 2012. Gastrotricha: A marine sister for a freshwater puzzle. PLoS ONE 7: e31740.

Received: 20 September 2013

Revised and accepted: 16 January 2014

Published online: 28 March 2014

Editor: R. Sluys 


\section{Appendix}

\section{Diagnosis}

Planodasyidae Rao and Clausen, 1970 (emended)

Elongate Macrodasyida, flattened ventrally and vaulted dorsally. Medium size terminal mouth opening. Head not clearly distinct from body. Cuticle naked. Pestle organs present or absent. Ventral cilia in two longitudinal rows, often united anteriorly. Epidermal glands variable in size and number. Anterior adhesive tubules in two diagonal rows, inserting directly on the cuticle; lateral adhesive tubules numerous; dorsal adhesive tubules present or absent; posterior tubules arranged on the margin of a single- or bilobed caudal end. Gonads paired; male anterior, female posterior; gametes maturing in a caudo-cephalic direction (Planodasys?); sperm ducts directed posteriorly, converging ventrally on the midline and opening into a common pore. Spermatozoon with peculiar prominent central sheath in the axoneme and basal region of the acrosome arranged as a straight tube with piled large oval vesicles. Caudal organ present; frontal organ usually present (Planodasys?). Intertidal or subtidal in distribution; fine to coarse sand. Type genus: Planodasys Rao and Clausen, 1970. Other genera: Crasiella Clausen, 1968; Megadasys Schmidt, 1974. 